\title{
Editorial: Neutrophils in Cancer
}

\author{
Brahm H. Segal ${ }^{1 *}$ and Zvi Fridlender ${ }^{2}$ \\ ${ }^{1}$ Roswell Park Comprehensive Cancer Center, Jacobs School of Medicine \& Biomedical Sciences, University at Buffalo, \\ Buffalo, NY, United States, 2 Institute of Pulmonary Medicine, Hadassah Medical Center, The Faculty of Medicine, Hebrew \\ University of Jerusalem, Jerusalem, Israel
}

Keywords: neutrophils (PMNs), cancer, tumor microenvironment, myeloid-derived suppressor cell (MDSC), innate immunity

\section{Editorial on the Research Topic}

Neutrophils in Cancer

\section{OPEN ACCESS}

Edited and reviewed by: Katy Rezvani,

University of Texas MD Anderson Cancer Center, United States

*Correspondence:

Brahm H. Segal

brahm.segal@roswellpark.org

Specialty section:

This article was submitted to

Cancer Immunity

and Immunotherapy,

a section of the journal

Frontiers in Immunology

Received: 25 January 2022

Accepted: 31 January 2022

Published: 18 February 2022

Citation:

Segal BH and Fridlender Z (2022)

Editorial: Neutrophils in Cancer.

Front. Immunol. 13:862257.

doi: 10.3389/fimmu.2022.862257
Neutrophils are short-lived immune cells whose main function is to mediate antimicrobial host defense. To function effectively, neutrophils must sense microbial products and host-derived cytokines and chemokines, traffic to sites of infection and injury, and kill pathogens. Neutrophils contain a broad armamentarium of antimicrobial products, many of which are sequestered in granules and activated by infection and mimics of infection (e.g. bacterial cell wall products). In addition to microbial products, neutrophils can be activated by damage-associated molecular patterns (DAMPs) (1-4), which are endogenous danger signals that are released from damaged or necrotic cells and activate the innate immune system by engaging pattern recognition receptors (PRRs). However, the same pathways that kill pathogens and amplify inflammation can also cause injury to the host. Neutrophil extracellular traps (NETs) are extracellular chromatin and granular constituents that are generated in response to microbial and damage motifs, and target extracellular pathogens, but are also pro-thrombotic and injurious (5). The research of the role of "Neutrophils in Cancer", the theme of this Research Topic, has emerged substantially during the last decade. A major theme of this Research Topic, relates to how antimicrobial pathways in neutrophils are activated by DAMPs and cytokine signals in the tumor microenvironment (TME) in ways that can promote or restrict tumor progression. Tumor-associated neutrophils are reprogrammed by cues in the TME, and, in turn, can cross-signal to tumor cells and reshape the immune landscape of the TME. These signaling interactions driven by neutrophils are potential targets for immunotherapy.

A number of studies show that tumor-infiltrating granulocytic cells are negative prognostic biomarkers. Gentles at al. (6) analyzed expression signatures from $\sim 18,000$ human tumors with overall survival outcomes across 39 malignancies, and identified intra-tumoral neutrophil signatures as the most significant adverse cancer-wide prognostic marker. Si et al. (7) noted intra-tumoral hotspots for co-localization of granulocytic and T cells in primary head and neck tumors, where a subset of granulocytes expressing LOX-1 and arginase I correlated with reduced activation of adjacent $\mathrm{T}$ cells.

The TME can influence neutrophil biology at multiple levels. Tumor-derived factors (e.g., G-CSF or GM-CSF) can drive abnormal myelopoiesis skewed toward granulocytic differentiation. Disordered myelopoiesis is a central mechanism for expansion of myeloid-derived suppressor cells (MDSC) in 
cancer. MDSCs are a heterogeneous subset of myeloid cells that can have a granulocytic or monocytic lineage, and are defined by their ability to suppress $\mathrm{T}$ cell immunity. The distinction between granulocytic MDSC (PMN-MDSC), which are typically defined by acquisition of suppressor function due to abnormal granulopoiesis (8), and normal neutrophils acquiring suppressor function in circulation or in the TME is important conceptually and therapeutically. One approach to limit PMN-MDSC expansion is based on the premise of tumor causing disordered granulopoiesis resulting in release of intrinsically suppressive granulocytes (8). Therapeutic targeting of neutrophils that acquire suppressor function after release from marrow would rely on impairing their trafficking to the TME [e.g., by CXCR2 inhibition (9)] and/or abrogating their suppressor function acquired in the TME.

One of the major themes of this Research Topic relates to neutrophils being recruited and altered by injurious and inflammatory cues in the TME. The similarity between tumor stroma and non-healing wounds has been recognized for decades (10). Normal wound healing following infection, trauma and other acute injuries is characterized by ordered stages of thrombosis, inflammation, and resolution. Neutrophils are the first responders to infection and injury; following resolution of the insult, inflammation transitions to less injurious chronic inflammatory cells (monocytes and lymphocytes). The TME is a pathologic, nonresolving wound characterized by persistent cellular injury and recruitment of mixed populations of inflammatory cells that comprise the immune landscape. Although neutrophil heterogeneity has been recognized for decades (11), the concept of distinct neutrophil populations at the transcriptome and phenotypic level has been advanced with molecular and cellular profiling tools. Neutrophils subsets in the TME can be differentiated at both the transcriptional (12) and metabolic levels (13), and the TME can induce distinct neutrophil clusters (14).

This neutrophil heterogeneity raises the notion for distinct populations accelerating or reducing tumor growth and metastasis. Neutrophils can bind to circulating tumor cells and enhance hematogenous metastasis through enhancing tumor cell cycle progression (15). Activated neutrophils can kill tumor cells through reactive oxidant generation $(16,17)$ and antibodydependent cellular cytotoxicity (ADCC). One exciting therapeutic approach involves enhancing ADCC of neutrophils and macrophages directed against tumor cells through inhibition of the SIRP $\alpha$-CD47 “don't eat me" pathway $(18,19)$. In contrast, tumor-infiltrating neutrophils can drive tumor progression and metastasis through a number of pathways, including stimulation of thrombosis and angiogenesis, stromal remodeling, and impairment of T cell-dependent anti-tumor immunity $(20,21)$. NETs can facilitate tumor progression in tumor-bearing mice

\section{REFERENCES}

1. Zhang Q, Raoof M, Chen Y, Sumi Y, Sursal T, Junger W, et al. Circulating Mitochondrial DAMPs Cause Inflammatory Responses to Injury. Nature (2010) 464(7285):104-7. doi: 10.1038/nature08780

2. Singel KL, Grzankowski KS, Khan A, Grimm MJ, D'Auria AC, Morrell K, et al. Mitochondrial DNA in the Tumour Microenvironment Activates through a number of mechanisms including vascular thrombosis, disabling of T cell immunity, and tumor cell signaling (22-24). Finally, although a substantial body of literature has shown that tumor-associated granulocytes can suppress $\mathrm{T}$ cell responses, different inflammatory or tumor-derived cues in the TME can prime neutrophil responses that enhance T-cell immunity $(25,26)$.

This Research Topic addresses key facets of how the TME influences granulocyte biology and how these distinct granulocyte populations influence tumor progression. Kramer and Abrams provide a cutting edge review of mechanisms driving PMN-MDSC generation and their suppressive effects on antitumor immunity. Furumaya et al. probe the concept of neutrophil plasticity in the TME, resulting in neutrophils exerting either pro-tumor or antitumor effects and discuss novel therapeutic approaches that target neutrophils. Hsu et al. provide a mechanistic overview of the specific steps where neutrophils can influence tumor growth and metastasis, including local tumor cell invasion and entry into circulation, survival of tumor cells and hematogenous metastatic seeding, and growth of tumor at metastatic sites. Zhang et al. specifically focused on the role of tumor-infiltrating N1 (antitumorigenic) and N2 (tumor-promoting, immunosuppressive) neutrophils in breast cancer. Domnich et al. review the role of salivary neutrophils in the development and progression of oral cancer. This review addresses neutrophils in the healthy oral cavity versus their activity in conditions that predispose to oral cancers (e.g., aging, smoking, and chronic periodontitis) and the effects of neutrophils in established oral cancers. Masucci et al. review the role of NETosis in tumor growth and metastasis, including their direct signaling to tumor cells, and highlight the potential application of NET inhibitors in cancer. The principles reviewed in this Research Topic provide an important mechanistic foundation for how neutrophils regulate the TME and for designing therapeutics to enhance tumor control, and add another important layer to our understanding of the immune TME in general, and specifically on the role of neutrophils in the progression of cancer.

\section{AUTHOR CONTRIBUTIONS}

Both authors contributed to the article and approved the submitted version.

\section{FUNDING}

\section{NIH R01CA188900-01 (BS).}

Neutrophils and is Associated With Worse Outcomes in Patients With Advanced Epithelial Ovarian Cancer. Br J Cancer (2019) 120(2):207-17. doi: 10.1038/s41416-018-0339-8

3. Schauer C, Janko C, Munoz LE, Zhao Y, Kienhofer D, Frey B, et al. Aggregated Neutrophil Extracellular Traps Limit Inflammation by Degrading Cytokines and Chemokines. Nat Med (2014) 20(5):511-7. doi: $10.1038 / \mathrm{nm} .3547$ 
4. Huebener P, Pradere JP, Hernandez C, Gwak GY, Caviglia JM, Mu X, et al. The HMGB1/RAGE Axis Triggers Neutrophil-Mediated Injury Amplification Following Necrosis. J Clin Invest (2015) 125(2):539-50. doi: 10.1172/JCI76887

5. Mutua V, Gershwin LJ. A Review of Neutrophil Extracellular Traps (NETs) in Disease: Potential Anti-NETs Therapeutics. Clin Rev Allergy Immunol (2021) 61(2):194-211. doi: 10.1007/s12016-020-08804-7

6. Gentles AJ, Newman AM, Liu CL, Bratman SV, Feng W, Kim D, et al. The Prognostic Landscape of Genes and Infiltrating Immune Cells Across Human Cancers. Nat Med (2015) 21(8):938-45. doi: 10.1038/nm.3909

7. Si Y, Merz SF, Jansen P, Wang B, Bruderek K, Altenhoff $P$, et al. Multidimensional Imaging Provides Evidence for Down-Regulation of $\mathrm{T}$ Cell Effector Function by MDSC in Human Cancer Tissue. Sci Immunol (2019) 4(40). doi: 10.1126/sciimmunol.aaw9159

8. Veglia F, Perego M, Gabrilovich D. Myeloid-Derived Suppressor Cells Coming of Age. Nat Immunol (2018) 19(2):108-19. doi: 10.1038/s41590017-0022-x

9. Nywening TM, Belt BA, Cullinan DR, Panni RZ, Han BJ, Sanford DE, et al. Targeting Both Tumour-Associated CXCR2(+) Neutrophils and CCR2(+) Macrophages Disrupts Myeloid Recruitment and Improves Chemotherapeutic Responses in Pancreatic Ductal Adenocarcinoma. Gut (2018) 67(6):1112-23. doi: 10.1136/gutjnl-2017-313738

10. Dvorak HF. Tumors: Wounds That do Not Heal. Similarities Between Tumor Stroma Generation and Wound Healing. N Engl J Med (1986) 315(26):16509. doi: 10.1056/NEJM198612253152606

11. Gallin JI. Human Neutrophil Heterogeneity Exists, But is it Meaningful? Blood (1984) 63(5):977-83. doi: 10.1182/blood.V63.5.977.bloodjournal635977

12. Shaul ME, Fridlender ZG. Tumour-Associated Neutrophils in Patients With Cancer. Nat Rev Clin Oncol (2019). doi: 10.1038/s41571-019-0222-4

13. Veglia F, Tyurin VA, Blasi M, De Leo A, Kossenkov AV, Donthireddy L, et al. Fatty Acid Transport Protein 2 Reprograms Neutrophils in Cancer. Nature (2019) 569(7754):73-8. doi: 10.1038/s41586-019-1118-2

14. Emmons TR, Giridharan T, Singel KL, Khan ANM, Ricciuti J, Howard K, et al. Mechanisms Driving Neutrophil-Induced T Cell Immunoparalysis in Ovarian Cancer. Cancer Immunol Res (2021). doi: 10.1158/2326-6066.CIR-20-0922

15. Szczerba BM, Castro-Giner F, Vetter M, Krol I, Gkountela S, Landin J, et al. Neutrophils Escort Circulating Tumour Cells to Enable Cell Cycle Progression. Nature (2019) 566(7745):553-7. doi: 10.1038/s41586-019-0915-y

16. Clark RA, Klebanoff SJ. Neutrophil-Mediated Tumor Cell Cytotoxicity: Role of the Peroxidase System. J Exp Med (1975) 141(6):1442-7. doi: 10.1084/ jem.141.6.1442

17. Clark RA, Klebanoff SJ. Role of the Myeloperoxidase-H2O2-Halide System in Concanavalin A- Induced Tumor Cell Killing by Human Neutrophils. J Immunol (1979) 122(6):2605-10.

18. Matlung HL, Babes L, Zhao XW, van Houdt M, Treffers LW, van Rees DJ, et al. Neutrophils Kill Antibody-Opsonized Cancer Cells by Trogoptosis. Cell Rep (2018) 23(13):3946-59 e6. doi: 10.1016/j.celrep.2018.05.082
19. Treffers LW, Zhao XW, van der Heijden J, Nagelkerke SQ, van Rees DJ, Gonzalez P, et al. Genetic Variation of Human Neutrophil Fcgamma Receptors and SIRPalpha in Antibody-Dependent Cellular Cytotoxicity Towards Cancer Cells. Eur J Immunol (2018) 48(2):344-54. doi: 10.1002/ eji.201747215

20. Singel KL, Segal BH. Neutrophils in the Tumor Microenvironment: Trying to Heal the Wound That Cannot Heal. Immunol Rev (2016) 273(1):329-43. doi: 10.1111/imr.12459

21. Singel KL, Emmons TR, Khan ANH, Mayor PC, Shen S, Wong JT, et al. Mature Neutrophils Suppress T Cell Immunity in Ovarian Cancer Microenvironment. JCI Insight (2019) 4(5). doi: 10.1172/jci.insight.122311

22. Cools-Lartigue J, Spicer J, McDonald B, Gowing S, Chow S, Giannias B, et al. Neutrophil Extracellular Traps Sequester Circulating Tumor Cells and Promote Metastasis. J Clin Invest (2013). doi: 10.1158/1538-7445.AM2012-2972

23. Lee W, Ko SY, Mohamed MS, Kenny HA, Lengyel E, Naora H. Neutrophils Facilitate Ovarian Cancer Premetastatic Niche Formation in the Omentum. J Exp Med (2019) 216(1):176-94. doi: 10.1084/jem.20181170

24. Albrengues J, Shields MA, Ng D, Park CG, Ambrico A, Poindexter ME, et al. Neutrophil Extracellular Traps Produced During Inflammation Awaken Dormant Cancer Cells in Mice. Science (2018) 361(6409). doi: 10.1126/ science.aao4227

25. Eruslanov EB, Bhojnagarwala PS, Quatromoni JG, Stephen TL, Ranganathan A, Deshpande C, et al. Tumor-Associated Neutrophils Stimulate T Cell Responses in Early-Stage Human Lung Cancer. J Clin Invest (2014) 124 (12):5466-80. doi: 10.1172/JCI77053

26. Singhal S, Bhojnagarwala PS, O'Brien S, Moon EK, Garfall AL, Rao AS, et al. Origin and Role of a Subset of Tumor-Associated Neutrophils With AntigenPresenting Cell Features in Early-Stage Human Lung Cancer. Cancer Cell (2016) 30(1):120-35. doi: 10.1016/j.ccell.2016.06.001

Conflict of Interest: The authors declare that the research was conducted in the absence of any commercial or financial relationships that could be construed as a potential conflict of interest.

Publisher's Note: All claims expressed in this article are solely those of the authors and do not necessarily represent those of their affiliated organizations, or those of the publisher, the editors and the reviewers. Any product that may be evaluated in this article, or claim that may be made by its manufacturer, is not guaranteed or endorsed by the publisher.

Copyright (C) 2022 Segal and Fridlender. This is an open-access article distributed under the terms of the Creative Commons Attribution License (CC BY). The use, distribution or reproduction in other forums is permitted, provided the original author(s) and the copyright owner(s) are credited and that the original publication in this journal is cited, in accordance with accepted academic practice. No use, distribution or reproduction is permitted which does not comply with these terms. 